\title{
POLÍTICAS SOCIAIS NA SAÚDE BUCAL - REVISÃO
}

\author{
SOCIAL POLICIES IN ORAL HEALTH - REVIEW
}

\section{Irene Fanny RITZEL ${ }^{1}$; Violeta Rodrigues AGUIAR ${ }^{2}$; Roger Keller CELESTE ${ }^{3}$}

\author{
${ }^{1}$ Universidade Federal do Rio Grande do Sul (UFRGS), \\ Faculdade de Odontologia, Programa de Pós-graduação \\ do Departamento de Saúde Bucal Coletiva, - Porto \\ Alegre, RS, Brasil. \\ ${ }^{2}$ Universidade Federal do Rio Grande do Sul (UFRGS), \\ Faculdade de Odontologia, Programa de Pós-graduação \\ do Departamento de Saúde Bucal Coletiva, - Porto \\ Alegre, RS, Brasil. \\ ${ }^{3}$ Universidade Federal do Rio Grande do Sul (UFRGS), \\ Faculdade de Odontologia, Departamento de Saúde \\ Bucal Coletiva, Porto Alegre, RS, Brasil.
}

Correspondência:

Nome: Irene Fanny Ritzel -

E-mail: irenefany@gmail.com

Endereço: Universidade Federal do Rio Grande do Sul

(UFRGS),

Faculdade de Odontologia - Programa de Pósgraduação em Odontologia

Rua Ramiros Barcels, 2492 - Porto Alegre - Rio Grande do Sul

CEP: 90035-003

\section{Resumo}

Sabe-se que as políticas sociais podem ter efeito importante sobre a pobreza e sobre as iniquidades socioeconômicas na saúde. Esta revisão de literatura tem o objetivo de descrever conceitos básicos sobre políticas sociais com enfoque em políticas de transferência de renda como direitos sociais. Por fim, discutem-se as influências de tais políticas na saúde bucal e seus potenciais mecanismos.

Palavras-chave: Saúde bucal. Políticas de saúde. Pobreza. Política social. Programas governamentais.

\footnotetext{
Abstract

Social policies can have an important effect on poverty and socioeconomic health inequities. This review has the objective of explaining basic concepts about social policies, with special attention to cash transfer programmes as social rights. Finally, it discussed the influences of social policies on oral health and its potential mechanisms.
}

Keywords: Oral health. Health policy. Poverty. Public policy. Government Programs. 


\section{INTRODUÇÃO}

As Políticas Públicas são a totalidade de ações, metas e planos que os governos (nacionais, estaduais ou municipais) traçam para alcançar o bem-estar da sociedade e o interesse público ${ }^{1}$, enquanto que políticas sociais são estratégias voltadas para proteção dos indivíduos e coletividade ${ }^{2}$. No processo de discussão, criação e execução das Políticas Públicas, encontramos basicamente dois tipos de atores: os 'estatais' (oriundos do Governo ou do Estado) e os 'privados' (oriundos da Sociedade Civil) ${ }^{1}$. As políticas de Welfare State - políticas de bem estar social, são consideradas de suma importância pois buscam ampliar os serviços e benefícios sociais à sociedade como um todo, mas especialmente proteger os grupos mais vulneráveis ${ }^{3}$, permitindo que as pessoas possam enfrentar situações que reduzem a capacidade de se inserirem no mercado de trabalho e produzirem seu sustento. Influenciam no bem estar individual, minimizando desemprego, pobreza, aposentadoria, doença e/ou invalidez, período de estudos, gestação $^{2,4}$.

Situações de pobreza e/ou miséria interferem decisivamente na elaboração de políticas públicas. A pobreza pode ser definida como privações contínuas de resources, capabilities, choices, security and power necessary for the enjoyment of an adequate standard of living and other civil, cultural, economic, political and social rights ${ }^{5}$. Caracteriza-se de forma ampla como fome, barreiras à educação formal, discriminação, vulnerabilidade e exclusão social ${ }^{6,7}$, bem como falta de segurança.

A presente revisão objetiva trazer conceitos relacionados às políticas públicas para a área de saúde bucal. Isto inclui o próprio conceito de políticas públicas, pobreza, transferência de renda. Ademais, o artigo busca descrever estudos recentes sobre o efeito que as políticas sociais podem ter sobre a saúde bucal. O texto está dividido em 5 partes, além da Introdução: Tipologias de Políticas Sociais, Políticas sociais nos Determinantes Sociais de Saúde, Direitos humanos e cidadania, Políticas de Transferência de Renda - Experiência brasileira e Experiências em outros países, e Efeitos das Políticas Sociais na Saúde Bucal.

\section{TIPOLOGIAS DAS POLÍTICAS SOCIAIS}

Criticas na concepção etnocêntrica sobre elaboração e operacionalização de políticas públicas podem levar a acreditar que a visão intervencionista parte do pressuposto de que há uma homogeneidade entre as pessoas, e o objetivo desta ação é o da homogeneização, ou seja, não trata os grupos sociais considerados "diferentes" como tais, mas na perspectiva de semelhança. Assim se explica a tendência das políticas públicas adotarem "modelos" de referência de sociedades consideradas "desenvolvidas". Ademais, a partir dessa concepção as necessidades dos grupos dominantes são absorvidas pelas sociedades consideradas pobres como suas, assim como a superação das carências da população pobre é feita utilizando-se de estratégias de grupos dominantes ${ }^{8,9}$.

O desenvolvimento teórico de políticas sociais, em especial no campo da sociologia, se deu bastante no pós-guerra europeu. Apesar das diferentes classificações das políticas sociais, há duas correntes clássicas expostas a seguir: a primeira corrente parte do conceito de políticas públicas com quatro divisões - distributivas, redistributivas, constitutivas e as regulatórias. As políticas distributivas agregam 
decisões tomadas pelo governo, nas quais a sociedade em geral financia e quem se beneficia são alguns estratos sociais ou grupos específicos. Como exemplo, podemos citar tanto a pavimentação e a iluminação de ruas quanto à oferta de equipamentos para deficientes físicos (como cadeiras de rodas). Nesse sentido, esse tipo de política não é universal, pois não é garantido por lei. Por outro lado, as políticas distributivas são de fácil implantação, porque raramente há opositores ao atendimento dessas demandas fragmentadas, pontuais e muitas vezes individuais ${ }^{10}$. Já as políticas redistributivas buscam redistribuir renda na forma de recursos e/ou de financiamento de equipamentos e serviços sociais. Os estratos sociais de alta renda financiam e os extratos sociais de baixa renda se beneficiam. Geralmente apresentam um elevado grau de conflito ${ }^{11}$. Há também as políticas chamadas regulatórias, que envolvem burocracia estatal e grupos de interesse na definição de ordens, proibições e regulamentações constitutivas. E as políticas constitutivas ou estruturadoras, que ditam as regras do jogo e definem as condições em que se aplicarão as políticas distributivas, redistributivas ou regulatórias ${ }^{10,11}$.

A segunda, classifica ${ }^{3}$ e descreve 3 modelos de políticas sociais para países capitalistas: residual, que se fundamenta na família e no mercado como provedores essenciais das necessidades humanas; meritocrática-particularista, para o qual a política social do Estado deve intervir temporariamente somente para corrigir distorções do mercado em uma lógica economicista; institucional-redistributivo, que pressupõe uma ação estatal para garantir proteção social para todos os cidadãos ${ }^{11}$.

As políticas públicas podem ser sociais: (saúde, assistência, habitação, educação, emprego, renda ou previdência), macroeconômicas (fiscal, monetária, cambial, industrial) e de outra natureza (científica e tecnológica, cultural, agrícola, agrária) ${ }^{11}$. Sabe-se que a pobreza é um grande problema no Brasil. E em geral relacionamos as políticas sociais a mecanismos que podem reduzir esse problema. Mas as políticas sociais são mais do que isso. Elas são parte constitutiva do Estado moderno desde o surgimento e desenvolvimento do capitalismo de tratamento das desigualdades geradas na própria sociedade, às quais requerem a intervenção estatal para a proteção social dos indivíduos e da coletividade ${ }^{3}$. E nesse âmbito a saúde tem um lugar importante ${ }^{2}$.

A saúde é uma necessidade humana básica. Embora se possa prevenir e evitar doenças, não é possível eliminá-las ao longo do curso de vida. Mas a saúde não está relacionada somente à ausência de doenças. Assim, num conceito amplo de saúde, esta se relaciona a situações que vão desde a segurança até a existência de uma renda razoável, uma habitação segura, um ambiente saudável. E isso na maioria das vezes não depende somente do indivíduo, mas de medidas sociais destinadas a toda a coletividade. Também, há situações que ameaçam a saúde e só podem ser evitadas e reduzidas por meio de ações coletivas. Exemplo disso são as medidas de controle sanitário, epidemiológico, a imunização contra doenças transmissíveis etc. Ou seja, a saúde, tanto como bem-estar quanto como prevenção de doenças, não é uma manifestação apenas individual e precisa ser tratada também no âmbito coletivo. Daí o papel do Estado e das políticas sociais na proteção à saúde dos cidadãos ${ }^{2}$.

As políticas públicas de saúde incluem aspectos como a regulamentação e licenciamento de prestadores de cuidados de saúde, o número de leitos hospitalares em 
uma comunidade, o regime de seguro e pagamento de serviços de saúde, a rede de serviços públicos e privados, a variedade, o tipo e qualidade dos serviços disponíveis na comunidade, e outras questões como o acesso e os custos dos serviços de saúde. Já políticas públicas "saudáveis" - ou para o desenvolvimento salutar, são as intersetoriais - como a educação ou transporte, e que podem ter influência importante sobre a saúde porque modificam conhecidos determinantes sociais em saúde ${ }^{12}$.

\section{POLÍTICAS SOCIAIS NOS DETERMINANTES SOCIAIS DE SAÚDE}

Determinantes sociais de saúde são políticas sociais que estão direta e indiretamente relacionados com promoção de saúde para indivíduos e comunidades ${ }^{13}$. A promoção de saúde fomenta a qualidade de vida através de pré-requisitos de recursos fundamentais: paz, habitação, educação, alimentação, renda, ecossistema estável, recursos sustentáveis, justiça social e equidade ${ }^{14}$.

Numa visão de coletividade, a saúde é determinada biologicamente - por fatores endógenos/biológicos, e socialmente por fatores exógenos/contexto de vida que resulta na produção social ${ }^{15}$. A intersetorialidade desses fatores se constituí nas políticas sociais de bem estar que atuam diferentemente nas sociedades, cada qual com suas necessidades ou escolhas com participação das comunidades ${ }^{13}$. Essas políticas sociais associadas com a distribuição de renda, combatem iniquidades na saúde para populações de baixa renda. A Bolsa Família (BF) é um exemplo de sinergismo social no Brasil através do Programa Bolsa Família (PBF) que faz coesão com outros programas governamentais, por exemplo, diminuindo mortalidade infantil ${ }^{16}$.

A partir dos determinantes sociais, econômicos e políticos são estruturados os determinantes sociais de saúde que se interagem e visam melhorias na qualidade de vida das pessoas. Interagem pelo nível micro - interações individuais, pelo nível médio comunidades, pelo macro - políticas públicas, até em ambientes de globalização. Temos fatores estruturais quando há conexão entre todos determinantes sociais, econômicos e políticos com fatores intermediários. Estes estão relacionados com as condições de vida e iniquidades na saúde ${ }^{17,18}$.

Os determinantes sociais de saúde influenciam na redução das desigualdades sociais, econômicas e de saúde das pessoas/populações. A desigualdade social é um fator de exclusão social associado à saúde, pobreza e cidadania no qual a renda per capita é um indicador socioeconômico que distingue países de alta renda e baixa renda $^{5,6,19}$. Associado a baixa renda, estão a miséria e pobreza e prejuízo da saúde ${ }^{20}$. A redistribuição de renda promovem redução e até erradicação da pobreza em países de média e baixa renda que impacta direta e indiretamente sobre a saúde dos povos ${ }^{7,21,22}$. Então é um fator coadjuvante de direitos humanos pois reduz inequidades sociais, minimiza desigualdades entre indivíduos e comunidades e promove o desenvolvimento dos países $^{13,17}$.

\section{DIREITOS HUMANOS E CIDADANIA}

Todo ser humano possui direitos sem distinção de sexo, etnia, credo, nacionalidade, idioma, liberdade de expressão e participação em assuntos políticos. Esses direitos se caracterizam por garantias legais nas esferas civil, política, econômica, social e cultural de todas populações democráticas ${ }^{23}$. Ao mesmo tempo em que o 
cidadão usufrui de direitos como de ir e vir e usa serviços e benefícios públicos, também não podem bloquear as vias públicas e tem o dever do pagamento dos impostos que subsidiam benefícios. Estes impostos e contribuições sociais são fundamentais para o Estado manter, por exemplo, a segurança pública e a defesa de direitos civis ${ }^{2}$.

Os direitos humanos no Brasil foram instituídos na Constituição de 1988 para defesa dos direitos e deveres dos cidadãos ${ }^{24}$. A cidadania interage indiretamente com saúde para a sobrevivência do cidadão, com economia que rege a distribuição das riquezas para promover saúde, e com política que rege distribuição das riquezas entre os cidadãos em diferentes nações ${ }^{6,24}$.

A cidadania se desenvolveu historicamente a partir de fases com conquistas crescentes $^{19}$. Iniciou com os direitos civis em meados do século XVIII referentes aos direitos à vida, segurança, liberdade e propriedades. Os direitos políticos foram constituídos apenas no século XIX correspondendo aos direitos à participação livre na política e eleições (votar e ser votado) de seus representantes no governo, muito embora tais direitos não incluíssem as mulheres em muitos países. No século $\mathrm{XX}$, tais conquistas incluíram direitos sociais que estão em consolidação e incluem direitos a necessidades básicas, salário mínimo digno, educação e saúde gratuita, habitação e alimentação que estão diretamente relacionados com a promoção de saúde do cidadão $^{19,20}$. Entre conquistas sociais estão as Transferências de Renda Continuada (TRC) e Transferência de Renda Não Continuada (TRNC) que garantem cidadania e diretos sociais recentes ainda não bem consolidados e alicerçados historicamente.

\section{POLÍTICAS DE TRANSFERÊNCIA DE RENDA}

A Transferência de Renda (TR) é uma política social de estratégia redistributiva de renda para cidadãos de países de baixa, média e alta renda. Iniciou no período do pós-guerra através do repasse em dinheiro direto ${ }^{25}$ e indireto, como por exemplo, os impostos para manutenção de serviços públicos pelo Estado ${ }^{26}$. Como política social, ela prioriza pessoas ou famílias pobres. Como os modelos adotados no Brasil: a BF que é uma TRC e o Benefício de Prestação Continuada (BPC) que é uma TRNC ${ }^{27}$.

Há dois tipos básicos de transferência de renda. A TRC é aquela modalidade que os beneficiários devem cumprir condicionalidades, normalmente voltadas para saúde, educação e/ou nutrição para o cidadão e membros da família, como o PBF no Brasil. Já a TRNC ocorre quando o beneficiário não tem obrigação de cumprir condições e está relacionada com a superação de pobreza da população como o BPC focado nos idosos e pessoas com deficiências físicas no Brasil $^{28}$.

A transferência de renda com condicionalidades ou sem condicionalidades visam a redistribuição de recursos econômicos para redução e até erradicação da pobreza. Há exemplos desse tipo de políticas em 171 países de alta, média e baixa renda - 52 são de TRC e 119 são de TRNC ${ }^{29}$.

\section{EXPERIÊNCIA BRASILEIRA}

Através do Programa Brasil Sem Miséria (PBSM) e criação do Cadastro Único cadastro socioeconômico para famílias brasileiras de baixa renda, o PBSM englobou programas com e sem condicionalidade para a superação da fome e miséria, acesso a serviços básicos de educação e saúde, geração de trabalho e renda, alfabetização de 
adultos, fornecimento de documentos e acesso ao crédito. O PBF é o maior e o mais importante programa social de redistribuição de renda do Brasil ${ }^{30}$ com focalização na pobreza $^{31}$.

As condicionalidades do PBF são direcionadas para crianças, adolescentes, gestantes e nutrizes para incentivar a demanda a serviços de saúde e educação ${ }^{32}$. As famílias devem cumprir o calendário de vacinas para crianças até sete anos, ações socioeducativas para adolescentes de até 15 anos retiradas do trabalho infantil, presença de $75 \%$ na escola para crianças e jovens de seis a 17 anos, acompanhamento pré-natal e saúde de nutrizes entre 14 e 44 anos de idade ${ }^{21}$.

O PBF atua como um coadjuvante no impacto social da saúde. Os efeitos do PBF são observados sobre alimentação e educação para as crianças e jovens. Tende a minimizar e dar resolutividade a doenças e está alterando a situação de pobreza no Brasil $^{33}$. Dentre as variáveis diretamente relacionadas com a saúde são observadas relações e efeitos com indicadores de mortalidade infantil ${ }^{16}$, acesso à serviços de saúde $^{34}$ e mais recentemente sobre a saúde bucal ${ }^{35}$.

O BPC é o principal programa não condicional que visa inserção do cidadão na sociedade brasileira focalizado em indivíduos com deficiência e idosos acima de 65 anos sem renda. Hoje, para receber este benefício, a renda familiar per capita deve ser menor que um quarto de salário mínimo. Esse direito foi assegurado na Constituição de 1988, e o efeito observado é o bem-estar a estes grupos e proteção contra a vulnerabilidade dos trabalhos informais ${ }^{28}$.

Os resultados até aqui evidenciados quanto a redistribuição de renda podem vir a ser modificados, desde inicio de políticas de austeridade fiscal em 2016, com a aprovação da Emenda Constitucional 95, ou PEC do fim do mundo, que congelou os investimentos públicos por 20 anos, e possivelmente expandidas com o novo governo em 2018. Houve alterações quanto a gestão municipal, estadual e federal, a confrontação de dados familiares, a prazos de inscrição, atualização e revalidação de dados, e a elegibilidade do beneficiário no Cadastro Único ${ }^{36}$. Ainda, o beneficiário passou a ter a responsabilidade de revalidar ou alterar os registros a cada dois anos o que pode levar a perda do benefício seja BF ou BPC.

O Decreto Federal n $n^{\circ}$ 9.674, de 2 de janeiro de 2019 transformou o MDS responsável nos governos anteriores pelo BF e BPC, em Secretaria Especial do Desenvolvimento Social (SEDS). O BF e o BPC permanecem na Secretaria SEDS no atual governo ${ }^{37}$. Até o momento, nenhuma mudança para os beneficiários foi instituída, no entanto, no plano de governos propõe um Programa de Renda Mínima para aprimorar o PBF, garantindo uma renda igual ou superior ao que é pago atualmente pelo BF - mas não ficou claro como isso seria realizado ${ }^{38}$. Apesar de aumentar o valor do benefício, o número de beneficiários pode reduzir, como já vem reduzindo.

\section{EXPERIÊNCIA EM OUTROS PAÍSES}

A estrutura das políticas sociais de cada pais é diferente. As TRC e TRNC objetivam diminuição da pobreza, aumento do capital humano, melhora na educação, saúde e nutrição que favorecem famílias e indivíduos beneficiados. $\mathrm{O}$ impacto financeiro é positivo e associado à segurança alimentar, aumento de despesas em alimentos e acesso à saúde das pessoas ${ }^{23}$. 
As primeiras ações tiveram início na Inglaterra em 1536 com a Lei dos Pobres, e primeiro programa de TR em $1795^{5}$. Vários países que erradicaram a pobreza absoluta e reduziram níveis de desigualdade fizeram uso de TR para parcela de pessoas que não conseguia a sua subsistência. Exemplos são Dinamarca - 1933, Estados Unidos - 1935, Inglaterra - 1948, Alemanha - 1961, Holanda - 1963, Bélgica - 1974, Irlanda - 1977, Luxemburgo - 1986, França - $1988^{39}$.

Na América Latina os principais programas sociais de TRC são direcionados aos grupos de crianças, gestantes e nutrizes: México, Equador, Peru, Colômbia e Chile ${ }^{25}$. As condicionalidades para adultos são menos frequentes e estão relacionadas com cuidados genéricos de saúde, acesso a medicações, testes de $\mathrm{HIV}^{23}$.

Quando comparado às políticas sociais dos programas do México e Chile estes são tão eficazes quanto o PBF, sendo que no Brasil há descentralização administrativa que diminui $\operatorname{custos}^{40}$. Os impactos que refletem na saúde são sobre o crescimento da criança, redução de anemia ${ }^{41}$, tuberculose, agressividade em crianças e desenvolvimento físico das mesmas ${ }^{41}$, desenvolvimento motor, cognitivo e linguagem ${ }^{23,41}$, baixa mortalidade infantil ${ }^{23}$.

Quanto a TRNC podem ser observados efeitos após períodos longos de sua implantação, em média de um a dois anos com melhora no bem estar em pessoas idosas com problemas mentais ${ }^{42}$, em anemia de mulheres ${ }^{43}$, e saúde, nutrição segurança alimentar e educação direcionadas à famílias que vivem na pobreza e vulnerabilidade ${ }^{26}$.

\section{EFEITOS DAS POLÍTICAS SOCIAIS NA SAÚDE BUCAL}

O principal suporte empírico em favor de políticas de redistribuição de renda reside na relação curvilínea entre renda e saúde, fenômeno também observado na saúde bucal $^{44,45}$. Assim, a redução de renda do grupo mais rico não impactaria muito na saúde bucal desse grupo, ao passo que o acréscimo ao grupo mais pobre seria relevante. Estudo longitudinal que avaliou mobilidade social sugere uma melhor saúde para o grupo em ascensão social do que para o grupo que permanece pobre ${ }^{46}$. Entretanto, a evidencia existente, mesmo em estudos longitudinais, utiliza desfechos prevalentes, dificultando-se as inferências causais, ou passíveis de viés de seleção.

São necessárias ações políticas que extrapolem os horizontes alcançados pela tecnologia e pelos conhecimentos específicos para dominar danos de reduzida complexidade como é o caso da cárie ${ }^{47}$. A defesa a universalização do acesso à água tratada e fluoretada deve continuar a ser uma das estratégias prioritárias da política nacional de saúde bucal ${ }^{48}$, por ser a melhor alternativa para cobertura em massa no campo da prevenção da cárie ${ }^{47}$.

O PBF - uma política social focalizada em população de baixa renda, pode ter relação com má higiene dentária e estar associado à cárie. Em 2010 na cidade de Pelotas /RS foi realizado um estudo com crianças de 8 a 12 anos de idade, estudantes de 20 escolas públicas e particulares. Os resultados demonstraram que a proporção de alunos beneficiados pela $\mathrm{BF}$ cresceu com o aumento da idade, com a menor renda familiar, menor escolaridade materna e com maior presença de placa dentária. Crianças de família não nuclear, que apresentavam CPOD $\geq 1$ e que nunca haviam feito uso de serviço odontológico na vida estiveram associadas ao recebimento do PBF. Dessa 
forma, escolares beneficiários do PBF possuem maior carga de cárie e são os que menos acessam os serviços odontológicos ${ }^{49}$.

Impactos na saúde bucal da vida cotidiana podem estar associados com educação e na falta desta, promover iniquidades em saúde bucal. Estudo em 21 países europeus que possuem regime de Welfare State comparou as iniquidades em saúde bucal e observou esta associação, isto é, a saúde bucal é perpassada pela educação ${ }^{50}$. Welfare State ou Estado de Bem-estar Social, pode ser compreendido como um conjunto de serviços e benefícios sociais de alcance universal promovidos pelo Estado $^{50}$ com a finalidade de garantir uma certa "harmonia" entre o avanço das forças de mercado e uma relativa estabilidade social. Também supri a sociedade de benefícios sociais como segurança aos indivíduos para manterem um mínimo de base material e níveis de padrão de vida, e para enfrentar os efeitos deletérios de uma estrutura de produção capitalista desenvolvida e excludente ${ }^{2,4}$.

Parece que a parte mais rica da sociedade beneficia-se mais de políticas sociais $^{51,52}$. Então políticas sociais de saúde bucal deveriam ser voltadas para toda população pois a abordagem individual apresenta limitações em estratégia de prevenção da cárie dentária. Isto é, quando a prevalência de cárie aumenta todo mundo aumenta a probabilidade de desenvolvimento de novas lesões de cárie, ou seja, o risco de desenvolvimento de uma lesão de cárie aumenta para todos os membros da população ${ }^{53}$.

Ademais, os países mais igualitários tendem a gastar mais em bens e serviços públicos. Além da maior coesão social e dos laços cooperativos que caracterizam o capital social, outras explicações para melhor nível de saúde em situações de menos desigualdade apontam as políticas públicas universalistas como responsáveis por parcela dos resultados ${ }^{54}$. Os modelos universais são apontados como mais eficientes (fazem mais com menos recursos), mais equânimes e, portanto, com maior impacto nas condições de saúde ${ }^{2}$. Apesar disso, novas intervenções e programas populacionais de saúde parecem atingir inicialmente aqueles de maior status socioeconômico, o que pode aumentar as iniquidades num primeiro momento, e só mais tarde beneficiar os indivíduos com maior vulnerabilidade social ${ }^{55}$.

\section{CONCLUSÃO}

Políticas introduzidas sem a necessária atenção para o problema social podem, muitas vezes, piorar a situação relativa de grupos de população submetidos à privação de direitos civis, econômicos, políticos e culturais. Programas de TR já estão consolidados em alguns países e em outros não, têm alcançado aparente melhora na qualidade de vida das pessoas.

As políticas públicas sociais foram a principal explicação sobre o efeito das desigualdades de renda sobre a saúde bucal. Por um lado, as políticas públicas parecem ter um efeito contextual importante para a maior parte da população, mas por outro, parece que fatores econômicos e sociais interagem na vida cotidiana das pessoas através de determinantes sociais de saúde fomentando saúde ou contribuindo para estruturar políticas voltadas para populações mais vulneráveis.

\section{Referências}


1. Lopes B, Amaral J. Políticas Públicas: conceitos e práticas. Caldas R, editor. Belo Horizonte: Sebrae; 2008. 48 p.

2. Lobato L de VC. Políticas socais e de saúde. Rio de Janeiro: CEBES; 2012. 73 p.

3. Titmuss RM. Essays on "The welfare state." POLICY PRESS; 1958. 232 p.

4. Gomes FG. Conflito social e welfare state: Estado e desenvolvimento social no Brasil. RAP. Rio de Janeiro; 2006;40(2):201-37.

5. Riachelis R. Esfera pública e Conselhos de Assistência Social: caminhos da construção democrática. 2nd ed. São Paulo: Cortez; 2000.

6. United Nations. The International Bill of Human Rights. Genova: General Assembly; 2003 p. 470.

7. United Nations. Economic and Social Council 2001/10. Genebra; 2001.

8. Rose G. Sick individuals and sick populations. Int J Epidemiol. 1985;14(1):32-8.

9. Boneti L. Políticas Públicas por Dentro. Ijuí: Unijií; 2011. 104 p.

10. Souza C. Políticas Públicas: uma revisão da literatura. Sociologias. 2006;8(16):20-45.

11. Moyses S, Goes P. A formulação de políticas públicas de saúde bucal. In: Planejamento, gestão e avaliação em saúde bucal. São Paulo: Artes Médicas; 2012. p. 248.

12. Donovan D, McDowell I. AFMC Primer on Population Health. 2nd ed. Ottawa: AFMC; 2017. 498 p.

13. Owusu-Addo E, Renzaho AMN, Smith BJS. Cash transfers and the social determinants of health: a conceptual framework. Heal Promot Int. 2018;1-13.

14. Organização Mundial da Saúde. Carta de Ottawa: primeira conferência internacional sobre promoção de saúde. In: Papeles de población. Otawa: OMS; 1986. p. 4.

15. Buss PM, Pellegrini Filho A. A Saúde e seus Determinantes Sociais. PHYSIS Rev Saúde Coletiva. 2007;17(1):77-93.

16. Rasella D, Aquino R, Santos CAT, Paes-Sousa R, Barreto ML. Effect of a conditional cash transfer programme on childhood mortality: a nationwide analysis of Brazilian municipalities. Lancet. 2013;382:57-64.

17. United Nations. Guia prático para a sociedade civil: o campo de ação da sociedade civil e o sistema dos direitos humanos das Nações Unidas. Genova: Organização das Nações Unidas (ONU); 2014. 32 p.

18. World Health Organization. All for equity: world conference on social determinants of health. Rio de Janeiro; 2012.

19. Marshall TH. Citizenship and social class: and other essays. London: Cambridge at the University Press; 1950. 128 p.

20. Arroyo MG. Educação e cidadania: quem educa o cidadão? 8th ed. São Paulo: Cortez; 2000.

21. Monnerat GL, De Castro M, Senna M, Schottz V, Magalhães R, Burlandy L, et al. Do direito incondicional à condicionalidade do direito: as contrapartidas do Programa Bolsa Família. Cien Saude Colet. 2007;12(6):1453-62.

22. Rego W. Aspéctos teóricos das políticas de cidadania: uma aproximação ao Bolsa Família. Lua Nova. São Paulo; 2008;73:147-85.

23. Walque D, Fernald L, Gertler P, Hidrobo M. Cash Transfers and Child and 
Adolescent Development. In: Child and Adolescent Health and Development. 3rd ed. Washington: International Bank for Reconstruction and Development, The World Bank; 2017. p. 482.

24. Owusu-Addo E, Cross R. The impact of conditional cash transfers on child health in low-and middle-income countries: a systematic review. Int J Public Heal. 2014;59:609-18.

25. Cecchini S, Atuesta B. Programas de transferencias condicionadas en América Latina y el Caribe: tendencias de cobertura e inversion. In: Series Politicas Sociales. Santiago: Naciones Unidas; 2017. p. 84.

26. Pega F, Liu SY, Walter S, Pabayo R, Saith R, Lhachimi SK. Unconditional cash transfers for reducing poverty and vulnerabilities : effect on use of health services and health outcomes in low- and middle-income countries ( Review ). Cochrane Library. 2017;(5):1-259.

27. Brasil., Ministério do Desenvolvimento Social e Combate à Fome, Secretaria Nacional de Renda de Cidadania. Coletânea da Legislação Básica do Cadastro Único e do Programa Bolsa Família Ministério do Desenvolvimento Social e Combate à Fome. 2nd ed. Brasília DF, editor. Brasília: Ministério de Desenvolvimento Social (MDS); 2015. 216 p.

28. Santos WR dos. Deficiência e BPC: o que muda na vida das pessoas atendidas? Cien Saude Colet. 2011;16(suppl 1):787-96.

29. Gentilini U, Honorat M, Yemtsov R. The State of Social Safety Nets 2014. 2014.

30. Cecchini S, Soares FV. Conditional cash transfers and health in Latin America. Lancet. Santiago: Published Online; 2015;385(4) Apr:32-4.

31. Rocha S. Transferências de renda federais: focalização e impactos sobre pobreza e desigualdade. R Econ Contemp. Rio de Janeiro; 2008;12(1):67-96.

32. Rocha S. O programa Bolsa Família: evolução e efeitos sobre a pobreza. Econ e Soc. 2011;20(1):113-39.

33. Correia LL, Rocha HAL, Leite ÁJM, Silva AC, Campos JS, Machado MMT, et al. The relation of cash transfer programs and food insecurity among families with preschool children living in semiarid climates in Brazil. Cad Saúde Coletiva. 2018;26(1):53-62.

34. Brasil. Inovações recentes no Programa Bolsa Família e impactos na superação da extrema pobreza. In: Painel técnico internacional de Programas de Transferência de Renda Condicionada na América Latina. Brasilia, DF: Ministério de Desenvolvimento Social (MDS); 2013. p. 21.

35. Calvasina P, Mota Pontes M, Barreto Oliveira J, G F Vieira-Meyer AP. The association of the Bolsa Familia Program with children's oral health in Brazil. BMC Public Health. 2018;18(1186):1-10.

36. Secretaria Nacional de Renda de Cidadania (Brasil). Sistema de gestão do Programa Bolsa Familia (SIGPBF). Brasilia; 2017.

37. Brasil. Decreto $N^{\circ}$ 9.667, de 2 de janeiro de 2019. Diário Of da União. 2019;

38. Meirelles H. Proposta de Plano de Governo Bolsonaro. 2018. p. 400.

39. Diniz S. Critérios de justiça e programas de renda mínina. Rev Katál Florianóp. 2007;10(1):105-14.

40. Helal DH, Neves JAB. Burocracia e inserção social: uma proposta para entender 
a gestão das organizações públicas no Brasil. Sociologias. Porto Alegre; 2010;12(25):312-40.

41. Ozer EJ, Fernald LCH, Manley JG, Gertler PJ. Effects of a conditional cash cransfer program on children's behavior problems what's known on this subject. Pediatrics. 2009;123:630-7.

42. Salinas-Rodríguez A, Torres-Pereda MDP, Manrique-Espinoza B, MorenoTamayo K, Solís MMTR. Impact of the non-contributory social pension program 70 y más on older adults' mental well-being. PLoS One. 2014;9(11):1-10.

43. Schady N. Cash transfers and anemia among women of reproductive age. Econ Lett. 2012;117(3):887-90.

44. Celeste RK, Oliveira SC, Junges R. Threshold-effect of income on periodontitis and interactions with race/ethnicity and education. Rev Bras Epidemiol. 2019;22.

45. Celeste RK, Nadanovsky P. Income and oral health relationship in Brazil: is there a threshold? Community Dent Oral Epidemiol. 2009;37:285-93.

46. Peres MA, Peres KG, Thomson WM, Broadbent JM, Gigante DP, Horta BL. The influence of family income trajectories from birth to adulthood on adult oral health: findings from the 1982 Pelotas birth cohort. Am J Public Heal. United States; 2011;101:730-6.

47. Pinto V. Bases para uma saúde bucal de caráter coletivo. 5th ed. São Paulo: Santos; 2005. 635 p.

48. Freire M do CM, Reis SCGB, Figueiredo N, Peres KG, Moreira R da S, Antunes JLF. Individual and contextual determinants of dental caries in Brazilian 12-yearolds in 2010. Rev Saúde Pública. 2013;47:1-10.

49. Oliveira LJC de, Correa MB, Nascimento GG, Goettems ML, Tarquínio SBCSBC, Torriani DDD, et al. Inequalities in oral health: are schoolchildren receiving the Bolsa Família more vulnerable? Rev Saúde Pública. 2013;47(6):1039-47.

50. Guarnizo-Herreno C, Watt R, Pikhart H, Sheiham A, Tsakos G. Socioeconomic inequalities in oral health in different European welfare state regimes. $\mathrm{J}$ Epidemiol community Heal. 2013;67(9):728-35.

51. Celeste RK, Nadanovsky P. How much of the income inequality effect can be explained by public policy? Evidence from oral health in Brazil. Health Policy (New York). 2010;97:250-8.

52. Celeste RK, Fritzell J, Nadanovsky P. The relationship between levels of income inequality and dental caries and periodontal diseases. Cad Saúde Pública. 2011;27(6):1111-20.

53. Batchelor P, Sheiham A. The limitations of a "high-risk" approach for the prevention of dental caries. Community Dent Oral Epidemiol. 2002;30:302-12.

54. Barata R.B. Como e por que as desigualdades sociais fazem mal à saúde. 21 st ed. Rio de Janeiro: FIOCRUZ; 2009. 120 p.

55. Victora CG, Vaughan JP, Barros FC, Silva AC, Tomasi E. Explaining trends in inequities: evidence from brazilian child health studies. Lancet. 2000;356:10938. 